\title{
Etude comparée de la quantité ingérée, de la digestibilité, de l'utilisation de l'azote, du temps moyen de rétention et du comportement alimentaire chez les jeunes caprins et ovins recevant différents régimes
}

\author{
C. MASSON, W. AlRAHMOUN (1), J.-L. TISSERAND \\ E.N.S.S.A.A., Laboratoire de recherches de la chaire de Zootechnie, \\ 26, boulevard Docteur-Petitjean, F 21100 Dijon
}

\begin{abstract}
Résumé
Sur trois boucs Alpin et trois béliers Ile de France castrés, âgés d'un an et pesant en moyenne $35 \mathrm{~kg}$ et $42 \mathrm{~kg}$ respectivement sont mesurés l'ingestibilité, la digestibilité, l'utilisation métabolique de l'azote, le temps moyen de rétention et le comportement alimentaire. Trois régimes sont successivement étudiés : foin de ray grass, paille traitée par la soude (4 p. 100) et paille traitée par la soude supplémentée de $100 \mathrm{~g}$ de lactose par jour.

La quantité de M.S. ingérée par $\mathrm{kg} \mathrm{p}^{0,75}$ est plus élevée chez les ovins que chez les caprins sauf pour la paille traitée + lactose. La paille traitée est moins ingestible que le foin chez les deux espèces.

La digestibilité de la matière sèche et la cellulose brute est supérieure chez les caprins à celle chez les ovins uniquement avec le régime paille traitée.

Le temps moyen de rétention des aliments dans l'ensemble du tube digestif est plus élevé chez les caprins, tandis que le temps moyen de rétention dans le réticulo-rumen est plus long chez les ovins.

La nature du régime modifie de façon importante le comportement alimentaire chez les deux espèces.

Avec les régimes à base de paille traitée, le bilan azoté est négatif chez les deux espèces. Cependant, la présence de lactose avec la paille traitée diminue nettement la teneur en urée du plasma et de l'urine chez les caprins. Il semble donc que le recyclage de l'urée sanguine soit plus intense chez les caprins que chez les ovins.
\end{abstract}

Mots clés : utilisation digestive, foin, paille, ovin, caprin.

\section{Introduction}

$\mathrm{Au}$ cours d'une expérimentation précédente (Alrahmoun W., Masson C. \& Tisserand J.L., 1985), nous avons mis en évidence, chez des animaux adultes

(1) Adresse actuelle : Université de Lattaquie, Faculté d’agronomie, Lattaquie, Syrie. 
recevant un régime carencé en azote, des différences importantes entre les caprins et les ovins en matière d'ingestion et d'activité microbienne dans le rumen. Celles-ci pourraient s'expliquer par un recyclage de l'urée plus intense chez les caprins que chez les ovins ainsi qu'un temps de rétention plus important dans le tube digestif, hypothèse déjà évoquée par Devendra (1981), Watson \& Norton (1982), Doyle \& Egan (1980) et Doyle, Egan \& Thalen (1984).

La présente étude réalisée avec des jeunes sujets, des espèces caprines et ovines, recevant des fourrages plus ou moins riches en azote, a pour objet de préciser l'importance de ce phénomène chez des jeunes animaux, l'âge des animaux étant un facteur de variation signalé par Brown \& JoHNSON (1984) dans leur revue bibliographique.

\section{Matériel et méthodes}

\section{A. Animaux}

Afin d'accentuer les réactions à la carence en azote nous avons choisi des jeunes animaux, castrés, âgés d'environ un an : trois béliers de race lle de France et trois boucs de race Alpine, pesant en moyenne 42 et $35 \mathrm{~kg}$ respectivement.

\section{B. Aliments}

Ces animaux reçoivent «ad libitum " pendant trois périodes successives de 6 semaines ( 3 semaines d'adaptation suivies de 3 semaines d'expérimentation) :

- un foin de ray grass,

- puis une paille traitée par la soude (semi-sèche à 4 p. 100),

- puis la même paille traitée complémentée avec $100 \mathrm{~g}$ de lactose par jour, dans le but de préciser l'effet d'un apport d'énergie rapidement fermentescible sur la teneur en urée du plasma et des urines.

La composition chimique de ces aliments est reportée dans le tableau 1.

Les fourrages et l'eau sont distribués en deux fois par jour ( $8 \mathrm{~h}$ et $17 \mathrm{~h}$ ), les quantités ingérées et la consommation d'eau sont contrôlées quotidiennement.

\section{Critères expérimentaux}

Nous avons retenu les critères expérimentaux suivants :

- ingestibilité, digestibilité et rétention azotée mesurées individuellement en cage à bilan sur une période de 10 jours ;

- cinétique de l'urée sanguine (prélèvements du sang jugulaire à jeun, $30 \mathrm{mn}$, $1 \mathrm{~h} 30,3 \mathrm{~h} 30$ et $6 \mathrm{~h} 30$ après le début du repas) et taux d'urée urinaire sur des échantillons récoltés le matin à jeun, ces deux mesures étant effectuées les jours qui précèdent et qui suivent la période de bilan digestif; 


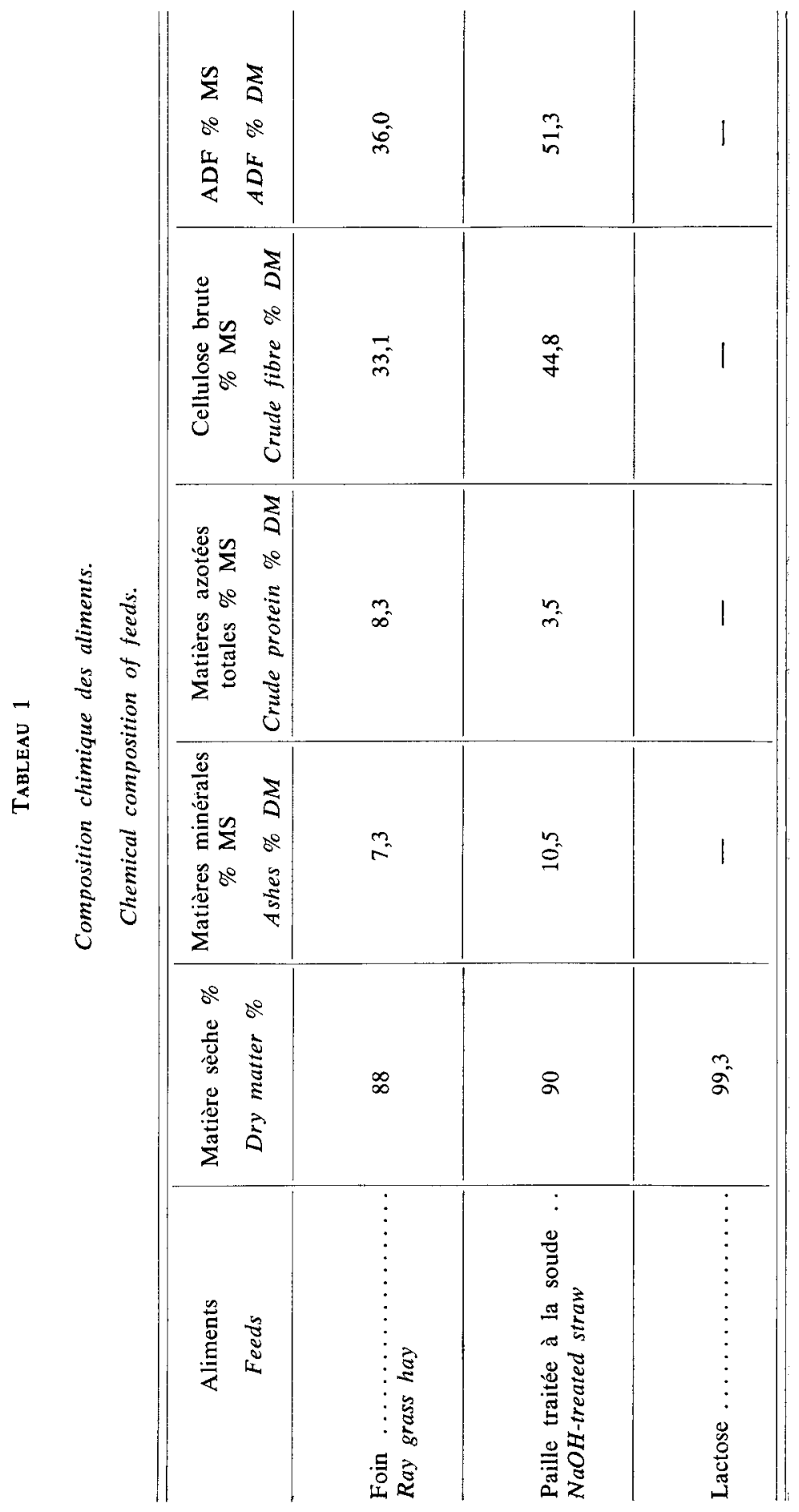


- enregistrement du comportement alimentaire (ingestion et rumination) sur deux boucs et deux moutons pendant trois jours consécutifs pour les régimes foin et "paille traitée ";

- évaluation de la vitesse de transit des particules pour les régimes de foin et de «paille traitée ». $30 \mathrm{~g}$ d'aliments mordancés (PONCET, 1985, communication personnelle) sont distribués à 8 heures le matin, les fèces sont récoltées selon un calendrier établi d'après les travaux de UDEN et al. (1982), c'est-à-dire 28 prélèvements de fèces entre la $8^{\circ}$ et la $144^{\circ}$ heure après la distribution; l'intervalle entre ces prélèvements étant de $2,4,4,4,8,12$ et $12 \mathrm{~h}$ respectivement du $1^{\text {er }}$ au $6^{\text {tu }}$ jour. La matière sèche de chaque échantillon est déterminée et les échantillons sont finement broyés pour y doser l'oxyde de chrome.

\section{Analyse}

Des prélèvements proportionnels des aliments distribués, des refus et des fèces de chaque animal sont séchés chaque jour dans une étuve ventilée à $80^{\circ} \mathrm{C}$ pendant 24 heures. Sur les échantillons cumulés, sur la période de 10 jours, sont déterminées les teneurs en matières minérales par incinération à $550^{\circ} \mathrm{C}$ pendant 6 heures, en cellulose brute selon la méthode de WEENDE et en matières azotées totales selon la méthode de KJELDAHL.

- Sur les fèces destinées à l'estimation du temps moyen de rétention, l'oxyde de chrome est dosé selon la technique décrite par FrançoIs et al. (1978) pour la minéralisation et Mathieson (1970) pour le dosage.

- Sur les urines, les teneurs en matières azotées totales (méthode KJELDAHL) sont dosées pour chaque animal et pour chaque période.

- Sur le plasma et l'urine, l'urée est dosée selon un test colorimétrique enzymatique (hydrolyse avec l'uréase).

- Le comportement alimentaire est enregistré à l'aide du vibrographe KIEnziETFW 3/8 décrit par RucKebusch \& Bueno (1973) pour les bovins et adapté aux petits ruminants par BECHET (1978).

\section{Résultats}

\section{A. Quantité ingérée (tabl. 2)}

La quantité de matière sèche ingérée $\left(\mathrm{g} / \mathrm{kg} \mathrm{P}^{0,75}\right)$ est statistiquement plus élevée $(P<0,05)$ chez les ovins que chez les caprins $(11$ p. 100 et 31 p. 100 pour les régimes «foin » et " paille traitée » respectivement), et avec le régime "paille traitée lactose », elle ne diffère pas entre les deux espèces.

Une chute importante (d'environ 45 p. 100) de la quantité de M.S. ingérée est notée chez les deux espèces lors du changement de régime (foin - paille).

\section{B. Digestibilité de la matière organique (tab1. 2)}

La digestibilité de la matière organique (M.O.) ne diffère pas statistiquement entre les deux espèces nourries au régime foin. Par contre, avec les régimes « paille», 


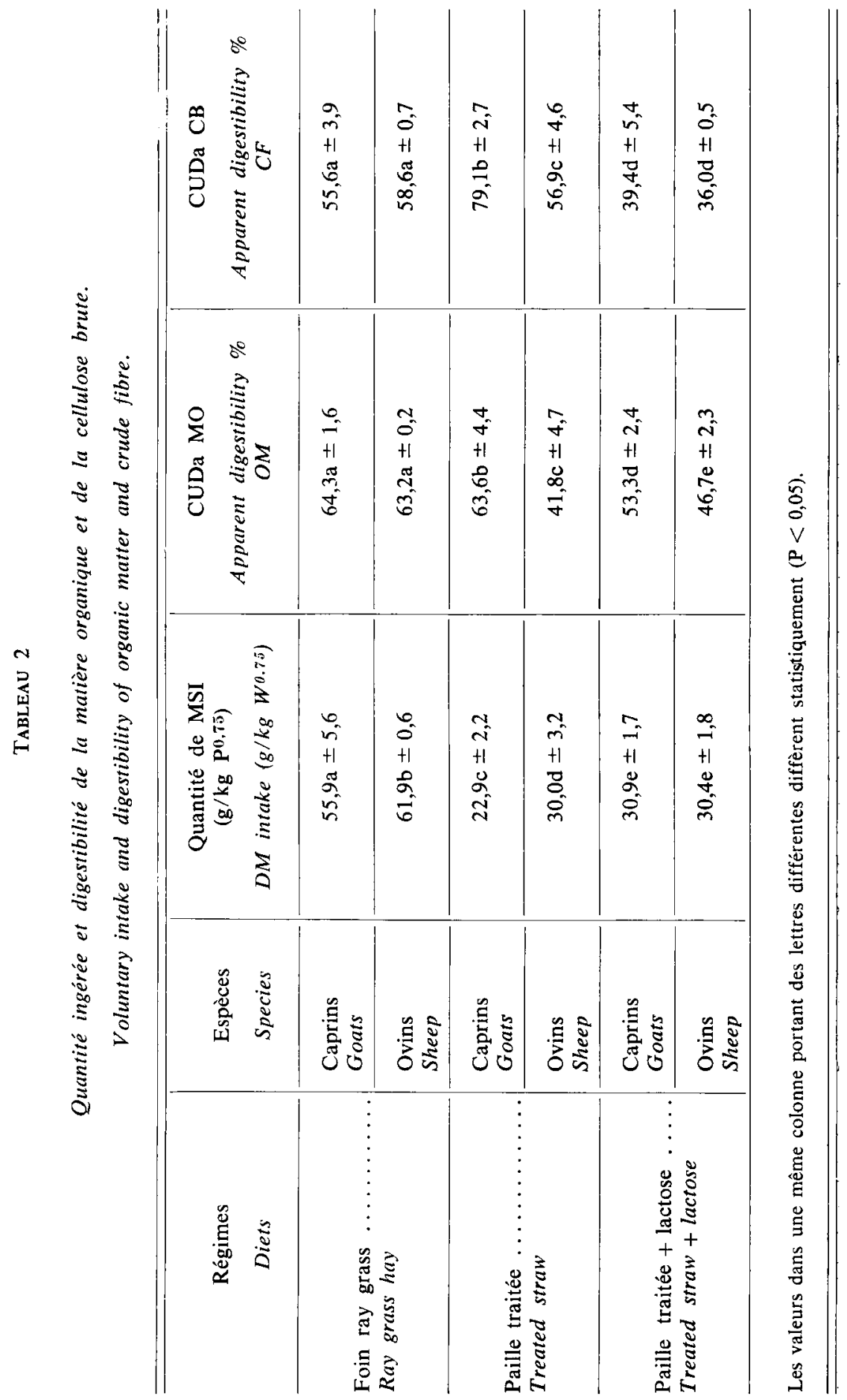


il existe des différences statistiquement significatives entre les deux espèces. Par rapport au régime "foin", cette digestibilité de la matière organique du régime « paille traitée " n'est pas modifiée chez les caprins, mais elle est fortement diminuée de 34 p. 100 chez les ovins. Par ailleurs, la complémentation en lactose diminue la digestibilité de la matière organique de la paille alors qu'elle l'augmente de 12 p. 100 chez les ovins.

\section{Digestibilité de la cellulose brute (tabl. 2)}

La digestibilité de la cellulose brute (C.B.) ne diffère pas statistiquement entre les deux espèces avec les régimes «foin» et "paille traitée lactose ". En revanche, avec le régime paille traitée, elle est nettement plus élevée chez les caprins (28 p. 100) que chez les ovins.

La complémentation de la paille traitée avec du lactose diminue la digestibilité de la cellulose brute chez les deux espèces (50 p. 100 chez les caprins et 37 p. 100 chez les ovins).

\section{Utilisation de l'azote (tabl. 3)}

La rétention de la matière azotée totale a tendance à être plus élevée chez les ovins que chez les caprins avec le régime «foin»; avec les régimes «pailles traitées $»$, elle est négative chez les deux espèces.

La teneur du plasma en urée (moyenne de 5 prélèvements journaliers), est plus élevée, quelle que soit l'espèce, avec le régime "foin " qu'avec les régimes "paille ". L'addition de lactose à la paille traitée entraîne une chute de l'urée plasmatique de 47 p. 100 chez les caprins et une légère diminution chez les ovins (7 p. 100).

La quantité d'urée urinaire est plus faible chez les caprins que chez les ovins quel que soit le régime (avec une différence de 42 p. 100, 27 p. 100 et 257 p. 100 pour les régimes : foin, paille traitée seule ou complémentée avec du lactose respectivement). Par rapport au régime «paille traitée seule », la complémentation de la paille traitée avec du lactose diminue la quantité d'urée urinaire, de 68 p. 100 chez les caprins et de $11 \mathrm{p}$. $100 \mathrm{chez}$ les ovins.

\section{E. Comportement alimentaire et temps moyen de rétention (tabl. 4)}

Alors qu'avec le régime de foin, la durée unitaire de mastication (ingestion + rumination) est plus faible chez les caprins que chez les ovins $(7,2$ contre $9,3 \mathrm{mn} / \mathrm{g}$ de M.S.I./kg $\mathbf{P}^{0,75}$ ), il n'y a plus de différence entre les deux espèces avec le régime paille traitée (de l'ordre de $15 \mathrm{mn} / \mathrm{g}$ de M.S.I./kg $\mathrm{P}^{0,75}$ ).

Le passage du foin à la paille traitée augmente la durée unitaire de mastication de 118 p. $100 \mathrm{chez}$ les caprins et de 64 p. $100 \mathrm{chez}$ les ovins.

Quel que soit le régime, le temps moyen de rétention effectué seulement sur deux animaux de chaque espèce, semble plus élevé chez les caprins que chez les ovins, et cela apparemment est d'autant plus vrai que le régime est riche en glucides pariétaux. Par contre, le temps moyen de rétention dans le réticulo-rumen semble plus élevé chez les ovins que chez les caprins. 


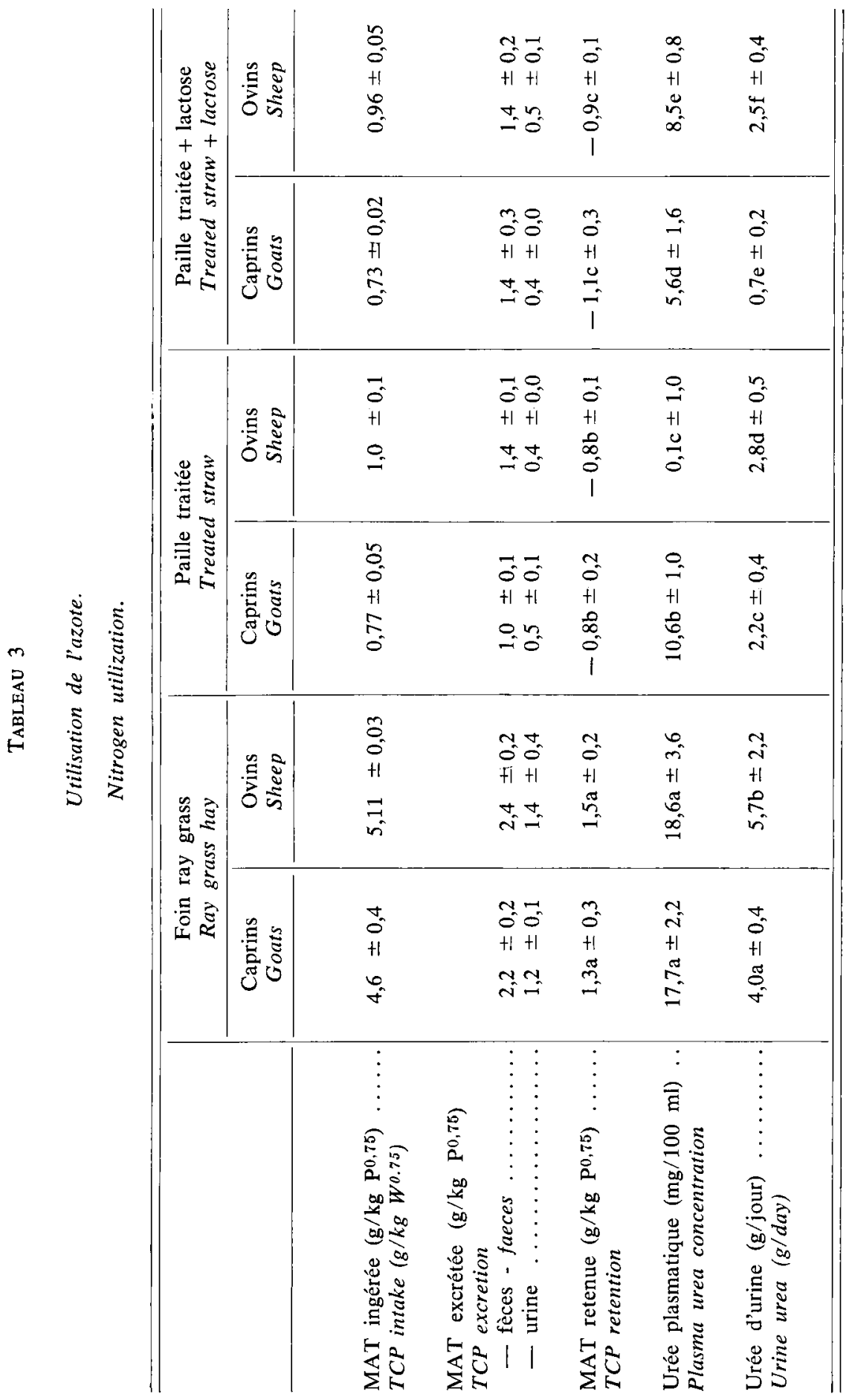


TABLEAU 4

Comportement alimentaire et temps moyen de rétention.

Feeding behaviour and mean retention time.

\begin{tabular}{|c|c|c|c|c|}
\hline & \multicolumn{2}{|c|}{$\begin{array}{l}\text { Foin ray grass } \\
\text { Ray grass hay }\end{array}$} & \multicolumn{2}{|c|}{$\begin{array}{l}\text { Paille traitée } \\
\text { Treated straw }\end{array}$} \\
\hline & $\begin{array}{l}\text { Caprins } \\
\text { Goats }\end{array}$ & $\begin{array}{l}\text { Ovins } \\
\text { Sheep }\end{array}$ & $\begin{array}{l}\text { Caprins } \\
\text { Goats }\end{array}$ & $\begin{array}{l}\text { Ovins } \\
\text { Sheep }\end{array}$ \\
\hline $\begin{array}{l}\text { Durée unitaire d'ingestion }(\mathrm{mn} / \mathrm{g} \text { de } \\
\left.\text { MSI } / \mathrm{kg} \mathbf{P}^{0,75}\right) \ldots \ldots \ldots \ldots \ldots \ldots \\
\text { Unitary eating time }\left(\min / \mathrm{g} / \mathrm{kg} W^{0.75}\right)\end{array}$ & $3,0 \pm 0,3$ & $4,0 \pm 0,4$ & $6,4 \pm 0,4$ & $4,4 \pm 1,1$ \\
\hline $\begin{array}{l}\text { Durée unitaire de rumination }(\mathrm{mn} / \mathrm{g} \\
\left.\quad \text { de } \mathrm{MSI} / \mathrm{kg} \mathrm{P}^{0,75}\right) \ldots \ldots \ldots \ldots \cdots \\
\text { Unitary ruminating time } \\
\quad\left(\text { min } / \mathrm{g} / \mathrm{kg} W^{0.75}\right)\end{array}$ & $4,2 \pm 0,5$ & $5,3 \pm 0,5$ & $9,3 \pm 1,0$ & $10,9 \pm 3,2$ \\
\hline $\begin{array}{l}\text { Temps moyen de rétention (h) } \ldots \\
\text { Mean retention time }\end{array}$ & 42 h 25 & 40 h 53 & 73 h 00 & 66 h 36 \\
\hline $\begin{array}{l}\text { Temps moyen de rétention dans le } \\
\text { réticulo-rumen } \ldots \ldots \ldots \ldots \ldots \ldots \\
\text { Mean retention time } \\
\text { in reticulo-rumen }\end{array}$ & 27 h 25 & 29 h 59 & $57 \mathrm{~h} 48$ & 61 h 44 \\
\hline
\end{tabular}

\section{Discussion et conclusion}

Cet essai réalisé avec un nombre limité d'animaux confirme les résultats de Brown \& JoHNSON (1984) qui rapportent que l'âge des animaux est un facteur important influençant la quantité de matière sèche ingérée chez les caprins et les ovins. En effet, les jeunes ovins consomment plus que les jeunes caprins, alors qu'Alrahmoun, Masson \& Tisserand (1985) ont observé le phénomène inverse avec des adultes. Il semble donc que chez les caprins, la capacité d'ingestion est plus importante comme le soulignent de nombreux auteurs, mais essentiellement chez les adultes.

Avec le régime de foin, la digestibilité de la matière organique et de la cellulose brute est peu différente entre les deux espèces. Ces observations sont en accord avec Wilson (1977), Blanchart, Brun-Bellut \& Vignon (1980), de Simiane et al. (1981), Alrahmoun, Masson \& Tisserand (1985). Avec le régime de paille traitée, la différence de la digestibilité de la matière organique et de la cellulose brute en faveur des caprins, est très importante. Ces résultats rejoignent ceux de Devendra (1974), Devendra \& Kuan (1979), Gihad (1976), Cuddeford \& DewaArd (1981) et Alrahmoun, Masson \& Tisserand (1985).

La présence de lactose dans la ration diminue la digestibilité de la cellulose brute chez les deux espèces, mais de façon plus importante chez les caprins. Il semble y avoir plusieurs explications possibles de cet effet négatif. Les glucides rapidement 
fermentescibles diminuent la digestion des glucides pariétaux, pour FuSEe \& LEATHERwood (1972) la synthèse de la cellulase par les Ruminococci est inhibée, et BERG (1975, 1978) observe une diminution de la production de la cellulase par certaines bactéries. Henning et al. (1980) notent un changement de la proportion des espèces bactériennes présentes dans le rumen. GuÉRIN \& DULPHY (1984) constatent une diminution de la digestibilité de la cellulose brute avec l'augmentation de l'énergie disponible de la ration.

Par ailleurs cette présence d'une source d'énergie disponible dans la ration influence le recyclage de l'urée. Selon Kennedy \& Milligan (1980), Harmeyer \& Martens (1980), l'addition de saccharose intensifie le recyclage de l'urée en augmentant le taux de transfert d'urée vers le rumen, ceci en diminuant la teneur en urée du plasma. Nos observations montrent que la présence de lactose dans la ration diminue nettement la teneur en urée plasmatique chez les caprins, tandis que celle des ovins est peu modifiée. Il semble donc que l'intensité du recyclage de l'urée chez les caprins est plus importante que chez les ovins. Cette possibilité de recyclage est corroborée par la réduction de la quantité d'urée urinaire, ceci en accord avec les résultats de ERIKsson \& VALTONEN (1982).

La durée unitaire d'ingestion du foin a tendance à être plus élevée chez les ovins, mais avec la paille traitée, elle est nettement plus importante chez les caprins. Ruckebusch \& Bost (1963), Geoffroy (1974), Focant (1984) enregistrent une durée unitaire d'ingestion plus importante chez les caprins avec des régimes à base de foin. Dans nos conditions expérimentales, la durée unitaire d'ingestion, plus élevée chez les caprins avec la paille traitée, pourrait être due à un comportement de tri différent chez les deux espèces. En effet, la paille refusée par les caprins contient plus de restes de limbes et moins de tiges que celle des ovins. De ce fait, le fourrage réellement ingéré par les caprins contient plus de tiges, soit plus de glucides pariétaux que chez les ovins, ce qui pourrait expliquer la durée unitaire d'ingestion plus élevée chez les caprins. Toutefois, ce comportement de tri des caprins rapporté par de nombreux auteurs, se fait en général en sens inverse, c'est-à-dire que les caprins ingèrent plus de feuilles que de tiges mais ces observations sont faites très souvent avec des fourrages plus caractéristiques tels que les légumineuses qu'avec de la paille où la distinction limbe-tige est assez délicate.

Le passage du régime foin au régime paille traitée, entraîne des augmentations parallèles de la durée de rumination et du temps moyen de rétention dans le réticulorumen. Ce prénomène, déjà signalé par Welch \& SMith (1969), et Welch (1982) est en relation avec le fait que la réduction des particules alimentaires jusqu'à une taille suffisamment petite pour passer à travers l'orifice réticulo-omasal est le résultat principalement de la rumination et de l'activité microbienne.

En conclusion, ces résultats confirment que la différence de digestibilité observée entre caprins et ovins apparaît importante avec les fourrages riches en cellulose et surtout pauvre en azote. La meilleure utilisation digestive des caprins peut s'expliquer par un recyclage plus intense de l'urée, la présence d'une source d'énergie rapidement disponible renforçant ce phénomène et par un temps moyen de rétention dans le tube digestif plus long. D'un autre côté, si l'âge des animaux doit être pris en compte dans la comparaison ovins-caprins, il semble plus important pour le niveau d'ingestion que l'utilisation digestive. En effet, les jeunes caprins semblent consommer moins que les jeunes ovins mais leur digestion est déjà plus efficace. 


\section{Summary \\ Comparative study of feed intake, digestibility, nitrogen utilization, mean retention time and feeding behaviour of young goats and sheep fed different diets}

Dry matter intake, digestibility, nitrogen utilization, mean retention time and feeding behaviour were compared in young castrated males (three Alpine goats and three Ile de France sheep). The animals weighed respectively 35 and $42 \mathrm{~kg}$. Three diets were successively studied : ray-grass hay, $\mathrm{NaOH}$-treated straw $(4 \mathrm{p} .100)$ and $\mathrm{NaOH}$-treated straw supplemented with $100 \mathrm{~g}$ lactose per day.

Dry matter intake $\left(\mathrm{g} / \mathrm{kg} \mathrm{W}^{0.75}\right)$ was higher in sheep than in goats with the diet including straw + lactose. Treated straw was less ingestible than hay in both species (table 2).

The mean retention time in the whole digestive tract was longer in goats than in sheep, whereas in the reticulo-rumen it was longer in sheep than in goats (table 4).

The nature of the diet strongly affected the feeding behaviour.

With the straw-treated diets, $\mathrm{N}$ balance was negative in both species, but the presence of lactose markedly reduced the plasma and urine urea concentration in goats. Plasma urea recycling seemed to be more important in goats than in sheep.

Key words : apparent digestibility, hay, straw, sheep, goat.

\section{Références bibliographiques}

alrahmoun W., Masson C., Tisserand J.L., 1986. Etude comparée de l'activité microbienne dans le rumen chez les caprins et les ovins. II. Effet du niveau azoté et de la nature de la source azotée. Ann. Zootech., 35.

BECHET G., 1978. Enregistrement des activités alimentaires et méryciques des ovins au pâturage. Ann. Zootech., 27, 107-113.

Berg B., 1975. Cellulase location in cellivibrio fulvus. Can. J. Microb., 21, 51-53.

Berg B., 1978. Cellulose degradation and cellulase formation by phialophora malorum. Archi. Microb., 118, 61-63.

Blanchart G., Brun-Bellut J., Vignon B., 1980. Comparaison des caprins alu ovins quant à l'ingestion, la digestibilité et la valeur alimentaire de diverses rations. Reprod. Nutr. Dévelop., 20, 1731-1732.

Brown L.E., Johnson L., 1984. Comparative intake and digestibility of forages and byproducts by goats and sheep. A review. Int. goat and sheep Res., 2, 212-226.

Cuddeford C., Dewaard T., 1981. Effect of urea supplementation on intake and utilization of a diet composed of whole barley and barley straw by immature goats and sheep. In : Symposium international nutrition et systèmes d'alimentation de la chèvre, vol. 1 , 160-164, éd. I.T.O.V.I.C.-INRA Tours, France, 545 pages.

De Simiane M., Giger S., Blanchart G., Huguet L., 1981. Valeur nutritionnelle et utilisation des fourrages cultivés intensivement. In : Symposium international nutrition et systèmes d'alimentation de la chèvre, vol. 1, 274-299, éd. I.T.O.V.I.C.-INRA Tours France.

DEVENDRA C., 1974. The intake and digestibility of napier grass (pennisetum purpureum) at four, five and six weeks of growth by goats and sheep in Trinidad. Turrialba, 25, 3.

Devendra $C_{\text {. }}$ 1981. The utilization of forages from cassava pigeon pea, leucaena and groundnut by goats and sheep in Malaysia, 338-345. In : Symposium international «Nutrition et systèmes d'alimentation de la chèvre», vol. 1. MORAND-Fehr P., Bourbouze A., DE Simiane M. ed. I.T.O.V.I.C., INRA-Tours, France. 
Devendra C., Kuan C.Y., 1979. The utilization of cultivated pigeon pea (cajanus cajan) forage by goats and sheep in Malaysia. Symposium on legumes in the tropics, 13-17 novembre 1979, Serdang, Selangor.

Doyle P.T., Egan J.K., 1980. Intake and digestion of herbage diets by Angora goats and Merino sheep. Proc. Aust. Soc. Anim. Prod., 13, $521-525$.

Doyle P.T., Egan J.K., Thalen A.J., 1984. Intake, digestion and nitrogen and sulfur retention in Angora goats and Merino sheep fed herbage diets. Aust. J. Exp. Agric. Anim. Husb., 24, 165-169.

Eriksson L., Valtonen M., 1982. Renal urea handling in goats fed high and low protein diets. J. Dairy Sci., 65, 385-389.

Focant M., 1984. Comportement alimentaire, rumination, fermentation réticulo-ruminale et acides gras volatils plasmatiques, comparés chez la chèvre et le mouton, influence du régime. Reprod. Nutr., Dévelop., 24, 239-250.

François E., Thill N., Thewis A., 1978. Méthode rapide de dosage de l'oxyde de chrome dans les aliments, les fèces et les contenus digestifs par titrage après oxydation nitroperchlorique. Ann. Zootech., 27, 355-361.

Fusee M.C., Leatherwood J.M., 1972. Regulation of cellulase form ruminococcus. Can. J. Microb., 18, 347-351.

Geoffroy F., 1974. Etude comparée du comportement alimentaire et merycique de deux petits ruminants : la chèvre et le mouton. Ann. Zootech., 23, 63-73.

GIHAD E.A., 1976. Intake, digestibility and nitrogen utilization of tropical natural grass hay by goats and sheep. J. Anim. Sci., 43, 879-883.

Guerin H., Dulphy J.P., 1984. Influence de l'apport complémentaire de mais, de pulpe de betterave ou de mélasse sur la valeur alimentaire d'un foin. Ann. Zootech., 33, 509-532.

Harmeyer J., Martens H., 1980. Aspects of urea metabolism in ruminants with reference to the goat. J. Dairy Sci., 63, 1707-1728.

Henning P.A., Vanderlinden Y., Matheyse M.E., Nauhaus W.K., Schwartz H.M., 1980. Factors affecting the intake on digestion of roughage by sheep fed maize straw supplements with maize grain. J. Agric. Sci. Camb., 94, 565-572.

Kennedy P.M., Milligan L.P., 1980. The degradation and utilization of endogenous urea in the gastro-intestinal tract of ruminants. A review. Can. J. Anim., Sci., 60, 205-221.

Matieson J., 1970. The automated estimation of chromic oxide. Proc. Nutr. Soc., 29, 30 A.

Ruckebusch Y., Bost J., 1963. Etude comparée de la motricité du réticulum et du comportement alimentaire chez les ovins et caprins en stabulation. Rev. Méd. Vét., 114, 184-196.

Ruckebusch Y., Bueno L., 1973. Un dispositif simple et autonome d'enregistrement de l'activité alimentaire chez les bovins au pâturage. Ann. Rech. Vét., 4, 627-636.

Uden P., Rounsaville T.R., Wiggans G.R., Van Soest P.J., 1982. The measurement of liquid and solid digesta retention in ruminants, equines and rabbits given timothy (phleum pratese) hay. Br. J. Nutr., 48, 329-339.

Watson C., Norton B.W., 1982. The utilization of pangola grass hay by sheep and Angora goats. Proc. Aust. Soc. Anim. Prod., 14, 467-470.

WELCH J.G., 1982. Rumination particle size and passage from the rumen. J. Anim. Sci., 54, 885-894.

Welch J.G., Sмiтh A.M., 1969. Influence of forage quality on rumination time in sheep. J. Anim. Sci, 28, 813-818.

WILson A.D., 1977. The digestibility and voluntary intake of the leaves of tree and shrubs by sheep and goats. Aust. J. Agric. Res., 28, 501-508. 